\title{
New developments and emerging trends in high-throughput screening methods for lead compound identification
}

This article was published in the following Dove Press journal:

International Journal of High Throughput Screening

5 August 2010

Number of times this article has been viewed

James W Noah

Drug Discovery Division, Southern Research Institute, Birmingham, Alabama, USA
Correspondence: James W Noah Southern Research Institute, 2000 9th Ave South, Birmingham, AL 35205, USA Tel $+\mathrm{I}-205-58 \mathrm{I}-2804$

Fax + I-205-58I-2726

Email j.noah@southernresearch.org

\begin{abstract}
High-throughput screening (HTS) campaigns have become a starting point for many drug discovery programs and are being conducted not only by large pharmaceutical companies but increasingly by smaller biotechnology companies, university research laboratories, and notfor-profit organizations. The underlying theme of any HTS campaign is the inventive application of brute force, and the pace and variety of invention strategies that have increased as HTS has evolved from a specialty capability to a standardized technology. Incorporation of HTS elements into the earliest phases of assay development supports the most economic and logistically feasible transition into a HTS campaign and subsequent lead compound discovery. There is a constant dilemma, however, when considering whether well-established, mature technologies should be replaced by new methods that promise to deliver spectacular economic, temporal, or logistical advantages. This is a review of some of the more novel and promising HTS technological and conceptual innovations that are currently being successfully used in the field.
\end{abstract}

Keywords: HTS, technologies, innovations, lead identification

\section{Introduction}

High-throughput screening (HTS) has not been the panacea for drug discovery that it was supposed to be. With the advent of HTS, the pharmaceutical industry was anticipating a rush of new potential drugs mined from the millions of uncharacterized small molecules held in chemical repositories. In much the same way that the magic bullets of interferon therapy or monoclonal antibodies missed their targets, HTS has not brought in a surge of new drugs. Drug discovery has matured enough to know that there is no single technique that will collapse the formidable barrier of finding new drug candidates. HTS, however, has proven to be a valuable and evolving technique that has greatly changed how we look at target and lead compound identification. Although the miniscule percentage of the lead compounds derived from an HTS campaign that actually achieve market status is often cited as the tolling of the proverbial bell for HTS, the secondary riches resulting from the technological advancement, standardization and commercialization of HTS reagents and products, and the continual redefining of what "high-throughput" means continue to motivate the industry to apply HTS to new assays every year. In addition, novel assay types and formats that deviate from the standard absorbance, fluorescent, or chemiluminescent endpoint methods have been developed to provide researchers with a great deal of choice when designing a primary HTS assay. Finally, the increases in the past decade in computational speed and data storage capacity have allowed vast amounts of imaging data to be collected 
and stored, meaning high-content HTS has become a reality. The review that follows looks at the motivation for, the development of, and the continuing evolution of novel HTS methods and assays.

\section{HTS evolution}

HTS was born with the invention of the microtiter plate by Dr Gyula Takatsky in 1951, who machined 6 rows of 12 "wells" in Lucite to make the first microtiter plate. ${ }^{1}$ The commercialization of a molded plastic version by John Liner (American Linbro) in the United States in the early 1950s made the microplate a standard item to be used in immunoassays. ${ }^{2}$ Although instrument readers for microplates were developed throughout the 1970s (primarily by Lab Systems) for the biomedical industry, the development of the laboratory-specific plate reader in 1981 by Biotek marked the broad adoption of the platform in a laboratory setting. ${ }^{3}$ By the 1990s, a myriad of pharmaceutical supply companies were producing microplates and instrument readers with different features, and by 2002, the demand for the standardization of microplates prompted the Society for Biomolecular Screening (SBS) to establish a Microplate Standards Working Group with the goal of publishing articles and information regarding SBS microtiter plate standards. ${ }^{4} \mathrm{~A}$ series of standard plate dimensions was proposed in 2003 and published by the American National Standards Institute on behalf of the SBS. Finally, the coevolution of both manual and automated liquid-handling systems occurred at the same time, beginning with the microtiter manual instrument, followed by the Autotiter automated system that was used extensively in the late 1960s and is the precursor for modern dispensers. ${ }^{5}$

Attempts at HTS assay development took place in the late 1970s using a combination of automation ${ }^{6}$ and continuousflow immunoassays ${ }^{7,8}$ to screen blood metabolite levels. These assays clearly describe the necessity for validation of parameters, such as coefficient of variance, day-to-day or operatorinduced variance, or inconsistencies due to reagents and materials, and suggest the development of laboratories that were specifically dedicated to HTS. The concept was not new, but since commercial automation was still sparsely available and expensive, early automation was often customized for the particular testing design and therefore not directly applicable to many different classes of assays or laboratory formats. The earliest examples of successful commercial assay automation can be found in the application of autosamplers, fraction collectors, and electronically-controlled pumps to the high-performance liquid chromatography arena. ${ }^{9}$ Continued advances in immunoassay systems also supported the movement to high-throughput formats, but the maturation of HTS automation and detection systems did not occur until the mid-1990s when a large increase in the number of plate formats, instrumentation, and subsequent publications of detailed HTS assay adaptations occurred. ${ }^{2}$ This evolution has continued, and very few modern screening institutions would still consider assays run in 96-well microplates as high-throughput compared with the 384-well and 1536-well microplate formats that are increasingly adopted. Below is a review of both innovative technologies that capitalize on modern HTS capabilities and innovative concepts about how HTS will continue to adapt to the needs of drug discovery programs.

\section{Innovative technologies}

Many HTS laboratories are recognizing that their future success is tied to viewing the entire drug discovery process as a whole and incorporating new technologies with an eye toward how these affect the entire process. Some factors in the decision to adopt new technologies are as follows: the value received for the incorporation of new techniques and resources versus miniaturization of the current process, the extent to which new technologies provide value that extends past the primary screening process, the information content provided by new technology, the technology's adaptability to multiple assay formats, and whether the new technology or instrument integrates into the laboratory while maintaining flexibility.

Several new technological innovations have been explored in the past decade, which have been designed not only to increase throughput (eg, miniaturization and combinatorial strategies) but also to increase the amount of data derived from a single assay point (multilabel screening and high-content screening [HCS] methods). These, coupled with increases in computational speed and capacity and evermore sophisticated and integrated bioinformatic programs, have increased the number and output of HTS laboratories dramatically in the same period. Finally, the adoption of new paradigm methods (such as label-free technologies) has encouraged the exploration of new targets and the expansion and consolidation of compound libraries to include a diverse range of target-focused molecules, natural products, and biological molecules. ${ }^{10}$ The following section discusses some of the technological innovations currently being used for HTS lead discovery, including assay miniaturization, target and endpoint selection, label-free applications, increasing chemical library diversity, continuing standardization for new assay formats, novel cell culture solutions, HCS, and applying HTS in infectious disease drug discovery. 


\section{Miniaturization}

Miniaturization is one of the most straightforward and most frequently applied strategies in current assay development. Cost, materials, time, and compound usage are the driving forces for the reduction of assay volumes, and such reduction can be most easily accomplished by reducing the size of microplate wells in the assay format although that does not always translate to a linear reduction in assay parameters. Miniaturization using high-density microtiter plates almost always requires a conversion to a homogeneous assay format in order to avoid the difficulties and, likely, the impossibility of manipulating submicroliter quantities of media during partition or washing operations. Miniaturization into the most efficient format is a crucial factor in increasing the number of screens performed per time and has proven to be one of the leading strategies that have been rapidly embraced and commercialized. Although miniaturization can introduce a new set of variables (acquisition and validation of new equipment for liquid handling, modification of cell-growth procedures because of differential surface-to-volume ratios, and the uniformity of temperature equilibration and ventilation of wells), the economic benefits and increased assay fidelity (fewer plate edge effects, lower plate critical variances) often experienced encourages the adaptation of the assay to the smallest workable volume. However, there are limits to how much an assay can be miniaturized; as cell-based assays are often restricted by the surface-to-volume ratio of plate wells. Problems such as uniform temperature equilibration, ventilation, and mitigating edge effects are the known caveats to any miniaturization strategy, and often creative solutions must be devised to overcome them.

The definition of HTS has changed during the last decade to accommodate the increasing throughput available by miniaturization of assays. Where the capacity for running a hundred 96-well plates might have been considered highthroughput a decade ago, it can hardly compete with the ultra-HTS systems that can assay several hundred 1536-well microplates daily. Since its invention, the microtiter plate has grown to include standardized 96-, 384-, 1536-well formats, with additional 3072-well nanoplate formats available for specialized or quantitative reverse-transcription polymerase chain reaction assays. ${ }^{11}$ As the line between microplates and nanoplates is crossed, HTS assay designs begin to resemble microarray formats, and this observation suggests future directions for HTS assay miniaturization strategies, as well as new directions for instrument configurations and capabilities.

\section{Target and endpoint selection}

Effective target selection sometimes means being the first to recognize important target characteristics and capitalize on them. Critical cellular constituents or pathways that are likely to be linked to an in vivo phenotypic response are typical targets. These can be either isolated targets that are organismspecific or a family of targets within a functional pathway that results in an ultimate downstream cellular response. Phenotypic responses include assays for cellular proliferation, death and environmental response apoptosis, cell-cycle control, DNA damage, or signal transduction pathway modulation. As the genomes of humans and pathogens are investigated in more detail, the target identification strategies are broadening their focus from the identification of novel targets to include the recharacterization of existing targets. Identification of important characteristics and implementation of subsequent discovery efforts make effective target selection the beginning of the drug discovery and assay development efforts. As the beginning, target selection is also the ideal phase at which to implement bioinformatics and support tools that can help plan and control the management of downstream processes of secondary assays, chemistry and cheminformatics, and lead compound selection.

Overall, the most important factor in the selection of a target and the accompanying assay is incorporation of physiological relevance into the assay design as early as possible. This means that both biochemical and cell-based assays are more often being coupled into a single-screen or counterscreen motif early in the process to demonstrate both compound target specificity and cellular efficacy. HTS is typically conducted with purified proteins or in a model cell system with an expressed molecular target, but the use and variety of cell-based assays in HTS have increased in the past 5 years. Because physiological relevance requires cell-based assays, the use of immortalized cells is a realistic concession, but primary cells are being considered more and more for HTS assays, which reflects the growing desire for the use of primary mammalian cells in HTS. In primary cells, the target is presumed to be expressed and function in an environment that more closely resembles that found in human diseases. ${ }^{12}$ Consequently, compounds screened in primary cell systems are likely to act in a more disease-characteristic fashion than in immortalized cells. Where the supply of primary cells (or in the case of human primary lines, the likelihood that these were compromised by isolation from patients with undesirable disease characteristics) was in the past a major bottleneck for HTS, the availability of 
cell lines derived from embryonic stems cells (ESCs) or tissues may be used to evaluate endogenous targets for drug discovery. Pluripotent ESCs can be grown much like immortalized cell lines and can be frozen in stock solutions or altered to express recombinant targets using viral vector systems. As the selection of targets continues to progress into the fields of protein-coupled receptors, ion channels, and kinase targets, it is becoming clearer that immortalized cell lines are limiting choices for physiologically-accurate assays, and the incorporation of stem cells represents a new and growing advancement for target relevance. ${ }^{12}$

\section{Label-free technologies}

Assays using label-free detection technologies have been available for more than a decade, but only in recent years those technologies have been adapted for HTS. Currently, the main use of label-free detection is for kinetics analysis, closely followed by affinity analysis. Most of these analyses are not in HTS formats but are instead done by secondary screening of selected hits and for lead optimization. In the past few years, new systems have been marketed that offer label-free screening for primary HTS assay applications. Assay possibilities using these new label-free tools include cell-based receptor or ligand interactions (particularly of orphan receptors), noninvasive cell proliferation and in vitro cytotoxicity testing, and antibody and small-molecule affinity analysis. Label-free detection technologies range from mass-spectrometry systems to reflectometric interference spectroscopy, measurement of dynamic mass redistribution (DMR) using waveguide resonance (Epic ${ }^{\circledR}$ System; Corning, NY, USA ${ }^{13}$ ), or surface plasmon resonance (Biacore ${ }^{\mathrm{TM}}$, GE Healthcare Lifesciences, Uppsala, Sweden ${ }^{14}$ ). Of these, only the latter 2 systems have evolved into commercially available instrument packages capable of limited or full HTS. Platform systems such as the Biacore have utilized a low-throughput label-free screening platform based on optical biosensor technology. The system performs both biochemical and cell-based drug discovery applications and offers drug developers the ability to rapidly evaluate promising new drug targets. It also allows for the observation of direct biological interactions not previously detectable in high-throughput applications. The Corning Epic System is an integrated high-throughput instrument and software system that is able to operate using specialized 384-well microplates. It uses a label-free and noninvasive method that is based on resonant waveguide grating biosensors. The resultant DMR signal serves as a functional optical signature for studying a variety of cellular target responses to compounds, is applicable to both biochemical and cell-based assays, and enables HTS using targets and assay methodology where the typical fluorescent or luminescent end points are not advised. This is still a new area of HTS advancement that is being explored by commercial entities, and much is expected in the future in the way of new instrumentation and detection packages for label-free assays.

\section{High-content screening}

Advances in bioinformatics software and increases in storage capacity, along with better automation and instrumentation, have allowed automated imaging to expand into the arena of cell-based HTS. This usage is called HCS. HCS is based on multiparametric approaches to multimicroscopic imaging at the single-cell level. It was conceived of as a secondary evaluation method to complement standard high-throughput primary screening in drug discovery and for validation of biochemical data, but since then HCS has expanded into the area of the life sciences as a method of simultaneously imaging a number of live cells and gathering unfiltered data using robotic fluorescent microscopy and automated image analysis. HCS is an innovative technology that has now become commercially available in complete instrument packages (currently available from at least 15 different commercial vendors) and has the potential to develop a true high-throughput capacity. Cellular imaging and analysis expands the tools used for drug discovery, allowing the mining of more physiologically-relevant data from biological systems using fully-automated microscopy. Examples of robust immunofluorescence-based assays, ${ }^{15}$ investigation of cell death and multiparameter cytoxicity screening, ${ }^{16}$ senescence, ${ }^{17}$ oncogenesis, ${ }^{18}$ stem cell proliferation, ${ }^{19}$ and pharmacokinetics ${ }^{20}$ are published. Finally, multiplexed HCS assays can be highly efficient tools for measuring the levels of multiple analytes in a single sample, provided the bioinformatics programs are able to integrate all of the data.

It should be noted that the HCS instrument is not an advanced plate reader, and the experiments must be designed so that valuable data can be extracted. This requires a deep understanding of the biology behind the systems and an understanding that subtle changes might be indicative of significant responses in cells. The throughput of an HCS instrument is a factor of the number of cells and fluorophores used. Also, because the analyses can take tens of minutes to 
completely image a single 1536-well plate (especially in the case of multiple fluorophores), it is important to note that large compound libraries can often be screened faster with other primary methods.

\section{Cell culture innovations}

One of the greatest sources of variability in HTS is the cell model itself. As stated above, physiological relevance requires at some point the integration of a cell-based model into every screening program to test drug efficacy and off-target effects. However, in the past, it has been extremely difficult to standardize cell preparations because subtle differences in passage, sources, or culture media components can result in amplified changes in assay performance. This causes HTS campaign bottlenecks that can significantly diminish the productivity of a drug discovery program. A new strategy designed to alleviate cell culture variation is the use of large batches of cryopreserved cells. These are cells that have been uniformly cultured in a single, large batch and then condensed and frozen at high cell densities and stored. When the assays are to be performed, the frozen cells are used to provide a uniform biological response throughout campaigns that last 3-4 months, without the variability that comes from multiple cell culture preparations. These methods have been used to support batch runs of several hundred 384-well plates of cells per day. ${ }^{21}$ It has been noted that cryopreserved cells in most cases work, but results are best when cell culture conditions match assay conditions because the cells are allowed only short times to acclimate to the assay conditions. Also, cryopreserved cells do not work for some assays. Therefore, comparisons should be made during each phase of assay validation to ensure that the assay maintains fidelity to the same assay using unfrozen cells.

A related and costly bottleneck within the drug discovery process is the creation of the modified cells needed for targeted HTS cell-based assays. This inability to transfect certain cell types uniformly or incorporate relevant agents (such as mRNA, proteins, and small molecules) into cell preparations often requires the use of model or surrogate systems that compromise assay physiological relevance. Because cells for HTS cell-based assays are commonly modified to express genes that produce proteins for drug targets, cell lines are traditionally modified for stable integration and expression of the target gene; this process can be long and time consuming, often taking several months to create a stable clone. A solution is to transfect cells with the target genes to produce a transient response for several days. Drug discovery groups are increasingly using transiently transfected cells because of the logistical savings versus the creation of stable cell lines. Common transient transfection techniques use lipid-based reagents or cell transduction with viral vectors, but both methods have limitations. Reagents for transfecting large volumes of cells in the preparation of an HTS scale campaign are expensive and not compatible with all cell types and loading agents. Limitations of viral vector-based transductions include the time and cost of vector production and scale-up, host antiviral responses to viral vectors that may skew the assay results, and a limited range of transducible cell types. To expand the range of cell and loading agent combinations and to overcome the limitations of other transient transfection methods, scalable flow electroporation technologies have been commercially developed (such as the MaxCyte ${ }^{\circledR}$ STX System, Gaithersburg, MD, USA ${ }^{22}$ ), which provide a growing availability of options in this niche.

Finally, automated cell culture systems are now available, which provide very meticulous cell culture handling and consistency in cell plating, and offer validated productivity and quality of cell lines without process change. In this case, each plate is handled identically in the assay with fixed intervals for all steps, even in multistep assays. The integration of robot handlers and high-performance readers is possible, providing fully automated systems.

\section{Continued standardization for new assay formats}

Many promising assay formats have been published in the past year, which are candidates for HTS development, including microfluidic chip-based approach to high-throughput drug screening ${ }^{23}$ and RNAi screens for whole-cell proteome surveys. ${ }^{24}$ Commercial HTS assay technologies for ion channel and receptor function are now available, and it is expected that the early standardization of these formats will mirror that with current microplate assays.

\section{Increased chemical library diversity}

The algorithm for an HTS campaign is an established and well-understood process that consists of a high-throughput primary screen (and likely a counterscreen), followed by a series of secondary assays that are target-specific, lower throughput, and designed to determine or confirm compound mechanism of action. These assay procedures are effective, but strategies to recognize hits that will eventually evolve into lead compounds comprise only half the battle. HTS laboratories 
are forced to deal with the major issue of compound library volume and composition that determines the scope and nature of their HTS programs. First, the quantities of compounds in company libraries are often quite small and of varying concentrations. Compound depletion is highly problematic, in most cases replacement is costly or extremely difficult to accomplish. Therefore, many laboratories also have a tendency to restrict the use of their libraries to specific types of screens, and all laboratories aim to maximize quantities of compounds in libraries and to minimize the quantities used in any given screen. The second issue is the diversity of current libraries. Many commercially-available libraries have large overlaps in diversity that result in duplication of efforts when whole library screens are performed in succession. Also, many of the compounds in these libraries are not like drugs. As a partial solution to this, a shift has been observed in drug discovery strategies during the past few years from simply identifying promising leads to simultaneously identifying dead-end leads that should be eliminated from consideration early in the screening process and tagged as possible false hits in future screens. This includes inherently toxic compounds, artifact-inducing compounds with a high sensitivity to assay conditions, or aggregate-promoting compounds that function by sequestering biomolecules. ${ }^{25}$ Although elimination of these compounds from libraries is advantageous from a screening position, it also further decreases library diversity. Continuing efforts to expand the available repertoire of chemical diversities are supported by several government programs, including the National Institutes of Health Molecular Libraries Program (NIH MLP) ${ }^{26}$ and the National Chemical Library within the National Center for Scientific Research to support the development of new methodologies for natural products chemistry. A key provision of this funding initiative is that the library designs are driven by biological rather than purely chemical considerations. In addition, since the goal is to identify effectors of truly novel biological phenotypes and mechanisms, the compounds produced under this initiative will represent chemotypes that are distinct from what is available commercially and in existing compound collections. ${ }^{26}$ Medicinal chemists have traditionally realized that increased chemical diversity, including natural products, will only support future HTS efforts. ${ }^{27}$

\section{Infectious disease screening}

One of the increasing uses of cell-based HTS is for broad antimicrobial drug discovery. Where a targeted approach has been traditionally used, many infectious disease screening programs optimistically apply large compound librar- ies to primary screening pathogen or cell-based models to simply measure a clear phenotype (eg, cytoprotection by the compound) and generate a hit list. The hits are then further characterized by counter, secondary or mechanistic assays to determine mode of action, target, and off-target effects. This method has the advantage, in that it requires only the unmodified model cell system to generate the primary hit list. However, HTS with live, infectious pathogens has only recently been adopted because of pathogen-specific biosafety perceptions and technological hurdles. Biosafety concerns include the exposure risk of working with any pathogen, which now frequently requires oversight by institutional biosafety committees and significant medical surveillance. The biosafety requirements also dictate the performance of many of the assay steps in a biosafety level 2 (BSL2) laboratory and in a biosafety cabinet. ${ }^{28}$ The evolution of liquid dispensers with integrated containment systems or minimized footprints is the direct result of the need for containment during pathogen screening. Other hurdles include the need to frequently decontaminate sensitive electronic equipment for routine preventative maintenance that often must be performed by qualified service representatives of the instrument manufacturers. This has prompted a growing population of service personnel who are not only versed in their instrumentation but also in broad infectious disease biosafety.

We will see this trend continue as BSL3 or BSL4 laboratories gradually acquire HTS capabilities. These capabilities will be of necessity of a modular nature and will consist of small footprint robots and readers that can be relocated and decontaminated reliably, and also use an increasing number of disposable, limited-use components. Although the need for biosafety and the space-limiting personal protective equipment will likely restrict the throughput of such laboratories (ie, it is quite likely that biocontained laboratories may never see the ultra-HTS performance levels that biochemical laboratories can achieve), HTS personnel will rapidly become accustomed to working in the biocontained environments necessary for screening a broad range of live pathogens.

\section{Innovative concepts}

With the increasing availability of instrumentation, bioinformatics software, and shared compound libraries, the paradigm of HTS is changing. Although only large pharmaceutical companies previously possessed both the financial muscle to purchase the expensive instrumentation and the large compound libraries that justified an HTS program, now smaller laboratories and individual researchers are incorporating 
HTS assay formats into newly developed assays with the direct intention of phasing these assays into large screening campaigns. This is more recently evident in academic settings, where the outsourcing for the screening of small, targeted libraries are becoming common. Academia has historically been involved in exploring the fundamental aspects of the disease targets and in developing tool molecules to better understand the genetic, biological, and biochemical basis of new and novel therapeutic targets. Academic laboratories have now entered the HTS arena, which has long been the domain of the pharmaceutical industry, with the understanding that the prospects of drug discovery are within their realm of investigation. This is coupled with a movement of experienced pharmaceutical drug discovery scientific talent into academia and the contracting of small molecule library synthesis to a number of university laboratories. It is clear that academia is poised to take new lead drug discovery to greater heights using HTS as a primary tool and taking advantage of the growing number of funding opportunities that have become available in the last 5 years that are designed to support cooperative lead compound discovery.

\section{New cooperative HTS initiatives}

One of the newest innovations for HTS is not technical at all but instead is a changing paradigm of HTS that is being increasingly adopted by academic and governmental institutions. The creation of government-funded programs such as the MLP (through the NIH Roadmap initiative) ${ }^{26}$ aims to enhance chemical biology efforts through HTS to obtain small-molecule tools (and ultimately, lead compounds) that are effective at modulating a defined biological process or disease state. The Molecular Libraries Probe Centers Network (MLPCN) ${ }^{26}$ is an example of a consortium of comprehensive and specialized HTS centers that perform biological assays submitted by the research community for screening against the Molecular Libraries Small Molecule Repository and also perform optimization chemistry on the hits to produce chemical tools and therapeutics. All of the results from the MLPCN's activities are placed into the public PubChem database,${ }^{29}$ and information about chemical probes is being made available to all researchers in both public and private sectors, for their use in studying biology and disease. Other newer programs include the National Cancer Institute's (NCI's) Chemical Biology Consortium (CBC), ${ }^{30}$ which is currently under development and will facilitate the discovery and development of new agents to treat cancer. It is again envisioned that this consortium will provide cutting-edge chemical tools for probing complex signaling pathways and will serve as the starting point for the discovery of targeted therapies. The mission of the CBC is to increase the flow of early stage drug candidates into NCI's drug development pipeline and to serve as an integrated research consortium at the interface of chemical biology and molecular oncology that will, working with various programs within the NCI, establish an iterative cancer drug discovery group on the scale of a small biotechnology company. ${ }^{30} \mathrm{~A}$ similar, nascent effort being formed is the National Toxicology Program (NTP), ${ }^{31}$ and a current program is being re-initiated by the National Institute for Neurological Disorders and Stroke (NINDS). ${ }^{32}$

By establishing a network of collaborating chemists, chemical and molecular biologists from government, industry, and academia, these new programs and their individual scientist participants can further address the unmet needs in therapeutic discovery by focusing on areas such as "undruggable" targets and underrepresented diseases or compound libraries (ie, natural products). Through the MLP, CBC, NTP, NCI, and NINDS programs and the interactions among the various participants, the drug discovery and development pipeline are enabled from target identification up to proof-of-concept preclinical trials. It is anticipated that participants will share in the intellectual property options that develop successful compounds created by these cooperative programs, but ultimately the early and rapid release of assay information and results to the public are expected to stimulate further research. The long-term vision of these collective programs is to bridge the gap between basic scientific investigation and clinical research supported by the NIH. This integrated process will allow NIH to make the world-class resources found in its participating institutions available to program participants.

\section{Summary}

The opportunity exists for great and rapid progress in HTS technologies and systems, with the recognition that this evolution is driven as much by economic and logistical realities as by the desire for increased information content. Chemical library diversity coupled with flexible instrumentation and adoption of innovative techniques are necessities, as is the simultaneous development of more sophisticated computational hardware and software to allow imaginative and novel ways of presenting and mining data. Integration of both technology and human components must occur throughout the entire drug discovery and development process. This means that the future HTS laboratory must have a broad range of 
expertise that includes economics, molecular biology, computer science, robotics, instrumentation, and overall process engineering and streamlining that considers current HTS practices for drug discovery and the evolving role of the HTS laboratory in biotech and pharmaceutical companies, academic centers, and institutes worldwide. There must also be a continual survey of the new HTS strategies to increase lead identification rates. Innovative strategies currently include the use of cell-based assays and high-content screening with infectious disease formats in biocontainment laboratories that use smaller footprint and modular equipment, as well as identification of targets and target classes of growing importance. Finally, the types of assays used and incorporation of new trends, including the use of primary or stem cells, miniaturization strategies to heighten throughput, increasing numbers and diversity of compounds screened, and the establishment of new cooperative HTS programs will directly address unmet needs in HTS and highlight the challenges and opportunities for HTS laboratories and researchers.

\section{Abbreviations}

HTS, high-throughput screening; qRT-PCR, quantitative reverse-transcription polymerase chain reaction; ESC, embryonic stem cells; DMR, dynamic mass redistribution; HCS, high-content screening; BSL, biosafety level; MLPCN, Molecular Libraries Probe Centers Network; CBC, Chemical Biology Consortium; NCI, National Cancer Institute; NIH, National Institutes of Health; NTP, National Toxicology Program; NINDS, National Institute for Neurological Disorders and Stroke; MLP, Molecular Libraries Program.

\section{Disclosure}

The author reports no conflict of interest in this work.

\section{References}

1. Ye S, Day INM. Microarrays and microplates: applications in biomedical sciences, 1st ed. London: Garland Science; 2003.

2. Mann RL. Microplate History 2nd ed. Society for Biomolecular Screening; 1999.

3. BioTek History Timeline. Winooski: Biotek.com. Available from: http:// www.biotek.com/about/timeline.html. Accessed May 17, 2010.

4. Published References Regarding Development of the SBS Microplate Standards. Chicago: Society of Biomolecular Sciences.com. Available from: http://www.sbsonline.com/msdc/pubrefs.php. Accessed May 17, 2010.

5. Evolution of Microplate Technology. Ontario: Labmanager.com. Available from http://www.labmanager.com/articles.asp?ID=458. Accessed May 17, 2010.
6. Holmes EW, Fareed J, Bermes EW Jr. Automation of plasma antithrombin III assays. Clin Chem. 1981;27(6):816-818.

7. Lloyd B, Burrin J, Smythe P, Alberti KG. Enzymic fluorometric continuous-flow assays for blood glucose, lactate, pyruvate, alanine, glycerol, and 3-hydroxybutyrate. Clin Chem. 1978;24(10):1724-1729.

8. Nolan JP, DiBenedetto G, Tarsa NJ. Continuous-flow enzyme immunoassay for thyroxine in serum. Clin Chem. 1981;27(5):738-741.

9. Dunnington DJ, Pinsky S, Mattes D, et al. Automation of data acquisition and processing in assays for anchorage-independent growth: application to the purification of epithelial transforming growth factor. Anal Biochem. 1988;174(1):257-264.

10. High Throughput Screening 2010 Study Finds HTS Laboratories Using Effective Screening Strategies. San Jose, CA: Business Wire. Available from: http://www.allbusiness.com/science-technology/chemistrypharmaceutical-chemistry-drug/14089966-1.html. Accessed Mar 10, 2010.

11. Brenan CJ, Roberts D, Hurley J. Nanoliter high-throughput PCR for DNA and RNA profiling. Methods Mol Biol. 2009;496:161-174.

12. Eglen RM. High throughput screening, high content screening, primary and stem cells new techniques now converging. Drug Discov World. Available from: http://www.ddw-online.com/screening/302933/ high_throughput_screening_high_content_screening_primary_and_ stem_cells.html. Accessed May 02, 2009.

13. Coring.com. Corning. Epic System. Available from http://www.corning. com/lifesciences/us_canada/en/whats_new/epic_system.aspx. Accessed May 07, 2010.

14. Biacore - Label-Free Interaction Analysis. Uppsala: GE Life Sciences. com. Available from: http://www.biacore.com/lifesciences/index.html. Accessed May 01, 2010.

15. Szafran AT, Szwarc M, Marcelli M, Mancini MA. Androgen receptor functional analyses by high throughput imaging: determination of ligand, cell cycle, and mutation-specific effects. PLoS One. 2008;3(11):e3605.

16. Rawlinson LA, O’Brien PJ, Brayden DJ. High content analysis of cytotoxic effects of pDMAEMA on human intestinal epithelial and monocyte cultures. J Control Release. Epub 2010 May 10.

17. Garavaglia A, Moiana A, Camnasio S, et al. Adaptation of NS cells growth and differentiation to high-throughput screening-compatible plates. BMC Neurosci. 2010;11:7.

18. Martinez ED, Dull AB, Beutler JA, Hager GL. High-content fluorescence-based screening for epigenetic modulators. Methods Enzymol. 2006;414:21-36.

19. Wright KT, Griffiths GJ, Johnson WE. A Comparison of High-Content Screening versus Manual Analysis to Assay the Effects of Mesenchymal Stem Cell-Conditioned Medium on Neurite Outgrowth In Vitro. J Biomol Screen. Epub 2010 Apr 16.

20. Christophe T, Jackson M, Jeon HK, et al. High content screening identifies decaprenyl-phosphoribose 2' epimerase as a target for intracellular antimycobacterial inhibitors. PLoS Pathog. 2009;5(10):e1000645.

21. Zaman GJ, de Roosa JA, Blomenröhra M, van Koppena CJ, Oosteroma J. Cryopreserved cells facilitate cell-based drug discovery. Drug Discov Today. 2007;12(13-14):521-526.

22. MaxCyte.com. Gaithersburg. MaxCyte Scalable Transfection System. Available from: http://www.maxcyte.com/cell-based-screening.shtml. Accessed May 14, 2010.

23. Thorsen TA. Microfluidic Tools for High-Throughput Screening. Biotechniques. 2004;36(2):197-199.

24. Karlas A, Machuy N, Shin Y, et al. Genome-wide RNAi screen identifies human host factors crucial for influenza virus replication. Nature. 2010;463(7282):818-822.

25. Shoichet BK. Screening in a spirit haunted world. Drug Discov Today. 2006;11(13-14):607-615.

26. MLI.gov. Washington DC. Molecular Libraries Program - pathways to discovery. Available from: http://mli.nih.gov/mli/. Accessed Apr 29, 2010.

27. Krier M, Bret G, Rognan D. Assessing the scaffold diversity of screening libraries. J Chem Inf Model. 2006;46(2):512-524. 
28. Jonsson CB, White L, Noah J, Severson W, Heil M. The Search for Promising Viral Targets in Drug Discovery. Amer Biotech Lab. 2007.

29. Wheeler DL, Barrett T, Benson DA, et al. Database resources of the National Center for Biotechnology Information. Nucleic Acids Res. 2007;35(Database issue):D5-D12.

30. Chemical Biology Consortium. Washington DC: NCI.gov. Available from: http://plan.cancer.gov/Chemical_Biology_Consortium.htm. Accessed May 12, 2010
31. National Toxicology Program High Throughput Screening Initiative. Available from: http://ntp.niehs.nih.gov/?objectid=05F80E15-F1F6975E-77DDEDBDF3B941CD. Accessed May 14, 2010.

32. Molecular Libraries and Imaging Roadmap Program. Washington DC: NINDS.gov. Available from: http://www.ninds.nih.gov/research/ molecular_libraries/index.htm. Accessed May 9, 2010.

\section{Publish your work in this journal}

International Journal of High Throughput Screening is an international, peer-reviewed, open access journal publishing original research, reports, editorials, reviews and commentaries dedicated to all aspects of high throughput screening, especially related to drug discovery and associated areas of biology and chemistry. The manuscript management sys- tem is completely online and includes a very quick and fair peer-review system. Visit http://www.dovepress.com/testimonials.php to read real quotes from published authors.

Submit your manuscript here: http://www.dovepress.com/international-journal-of-high-throughput-screening-journal 